\title{
Infrared diagnostics of blistering disease of cheeks and lips
}

\author{
by A. Reshetnikov*, M. Kopylov*, A. Urakov*, T. Urakova* \\ * Izhevsk State Medical Academy, 281, Kommunarov Str., Izhevsk, Russia, urakoval@live.ru
}

\begin{abstract}
In order to ensure early diagnostics of iatrogenic injuries to oral cavity soft tissues occurring as a result of dental treatment, we propose to control the dynamics of the local temperature of interior lips and cheeks surface in patients after undergoing dental procedures (after medicine injections, due to pushing effect of surgical instruments, after installation of dental crowns, implants and dentures), and especially after the installation of braces. Using thermal imaging camera to detect local hyperthermia areas observed at the sites of injections and due to the instrumental and/or technological manipulations is proved to be a promising and versatile test for assessing the aggressive impact of these manipulations, how the patients adapt to them and especially to the brackets. We developed a new method for infrared diagnostics of the disease called "blistering disease of lips and cheeks".
\end{abstract}

Keywords: temperature, blisters, ulcers, inflammation, soft tissue injury, dental care, braces.

\section{Introduction}

During dental treatment, doctors often inject proper medicines (tablets and solutions for injection) and put surgical and diagnostic instruments into patients' oral cavity, which may sometimes cause local injuries and damage the oral cavity soft tissues [1-5]. Besides, soft tissues in patients' oral cavity may be damaged by installed dental appliances: crowns, dentures, fake teeth and braces [6-8]. Moreover, the soft tissue injuries in patients' mouth and face are not always visible, may not occur immediately after dental treatment, and thus may not be diagnosed immediately. Sometimes iatrogenic soft tissue injuries occur in patients without being noticed by them and dentists. Sometimes iatrogenic injuries of the oral cavity soft tissues in patients develop a few hours or days after their visit to the dentist. At present, such injuries are not detected and diagnosed in a timely manner.

Because soft tissue injuries caused by dental treatment refer to iatrogenic damages, their occurrence is troublesome not only for patients but for dentist as well. In this context, it becomes clear that hidden or invisible soft tissue injuries may cause the greatest troubles, as they appear far away from the dentist and may end with serious complications.

Visible iatrogenic injuries of oral cavity soft tissues include abrasions, bruises and the abscess in face and mucous membranes of the lips, gums, tongue, cheeks [9]. The most visible iatrogenic injuries in the face are bruises. Medicine injections and too strong and long-lasting soft tissue compression by surgical instruments are the most common reasons for bruising $[6,8]$. Facial bruises and other visible iatrogenic injuries impair the aesthetic result of dental treatment. However bruising is not considered as a disease itself as it often proceeds without local inflammation and does not end with necrosis and abscesses [10].

Invisible iatrogenic injuries to the soft tissues of the face and mouth include local inflammations, which cause nonthreatening reversible damage to tissues. However, sometimes the inflammatory process may become irreversible, and then after several days a blister may appear at the site of reversible tissue damage, and may later turn into the mucosal ulcer [2].

It is established that certain surgical and diagnostic instruments used by dentists, as well as some dental appliances and products produce multiple invisible damages to the soft tissues that often cause their reversible inflammatory injuries [11-14]. Braces are leaders in producing multiple local damages to the patients' lips and cheeks, and they can cause blisters and sores. So, it may be no accident that brackets splinting is suggested in order to reduce their damaging effect [9].

Today, in scientific literature, blisters, sores and ulcers are considered as direct results from soft tissue injuries. However, until now there is no detailed description of the etiology, pathogenesis, and the outcomes of lips and cheeks blistering disease caused by the installment of braces and other dental appliances. There is also no standard for diagnosis, prevention and treatment of this new disease. Until now, the difference between the progress of blistering disease in lips from its progress in cheeks is not clear. Moreover, currently there is only one study on the mouth ulceration caused by brackets [9]. Furthermore, until now the relation between the mucosa color change and its temperature dynamics in ulcerative and nonulcerative closed soft tissue injuries has not been studied. Besides, until now the relationship between inflammation, hemorrhage, blisters and ulceration of soft tissue of cheeks and lips inner surface is not clarified, in particular, when they are caused by dental tools and appliances.

It is expected that studying the dynamics of local temperature in patients' facial skin and oral cavity mucous membranes after completing their dental treatment, and especially after installation of dental appliances with rough and uneven surface will avert iatrogenic disease and/or provide its early diagnosis as well as prevent irreversible inflammatory soft tissue damage [15]. 


\section{Materials and methods}

During Healthy adult volunteers (Group $1, \mathrm{n}=10$ ) with body mass of $55-72 \mathrm{~kg}$ and adult patients with brackets (Group 2, $\mathrm{n}=30$ ) with body mass of $48-63 \mathrm{~kg}$ were observed during 2009-2016. We obtained thermal images of the subjects' facial skin and mucous membranes of the lips, cheeks, gums and tongue immediately after installing the braces and then every day during one week. All tested patients were standing upright. Infrared monitoring was performed with ThermoTracer TH9100XX (NEC, USA) thermal imager. Thermal imager was installed at a distance of 1 meter from the surface of the shield. Ambient temperature in the examination room was $24-25^{\circ} \mathrm{C}$, the temperature field of the thermal camera was set in the range of 25 to $36^{\circ} \mathrm{C}$. The data obtained were processed using the Thermography Explorer and Image Processor software. The photographs were archived in the form of thermomaps atlas. Before the beginning of the research, its protocol was approved by the Ethics Committee of "Resto" dental clinic (Izhevsk), based on the principles set in the Declaration of Helsinki of the World Health Organization. All patients involved signed an informed consent for participation in the study.

\section{Results}

The results of our study confirmed that modern braces present the major hazard for the mucous membranes of patient's lips and cheeks. Multiple local irritations, inflammations and necrotic damages to the inner surface of cheeks and lips were observed in 7 from 30 patients after installation of brackets (Figure 1). It is shown that local inflammations occur in the beginning, then they disappear or get worse, transforming into watery blisters, and later into sores that scar after 2-3 weeks. Local inflammations occur during the first week after brackets installation; patients discover them because of painful sensation in their cheek and/or lip. Besides, the pain and the severity of soft tissues local irritation caused by brackets increases while chewing the food. It has been found that if a patient does not observe local irritations, cheeks and lips injuries in one week after the brackets installation, these injuries will not appear later. It is also found that after successful regeneration of damaged tissues, recurrent injuries of lips and cheeks are also not likely to occur.

We were able to establish that local irritations in lips and cheeks result from prolonged, intensive and continuous chewing movements of the patient's jaw. At the same time local irritations appeared in 2 days after braces installation in one patient, after 3 days in 2 patients, and after 4 days in 4 patients. Herein, cheeks were damaged earlier and more often than lips. Thus, in all 7 patients in this group braces caused local damage to their cheeks and only in 4 patients in this group braces caused further damage to the inner surface of the lips.

It appeared that multiple local injuries of cheeks and lips in 4 of 7 patients were localized on one side. Moreover, local injuries of lips and cheeks appeared on that side of the mouth, where patient mostly chewed food. First, irritation sites appeared as the zones of hyperemia, hyperthermia and itching, and later, within 1-2 days they transformed into local injuries, taking the form of watery bubbles (Figure 2). These bubbles are similar to blisters that occur on the hands and feet skin because of excessively strong and forceful rubbing of the skin. It is noteworthy that each blister was located opposite the projecting bracket part, installed on the surface of the nearest tooth. The location of the zones of mucous membranes local irritation, inflammation, blistering and erosion on either side of the median face line was observed less often. This type of localization of soft tissues irritation and inflammation zones caused by braces was observed in patients who chewed food equally using the left and right sides of their masticatory apparatus.

Analysis of patients' questionnaire responses showed that the damaging effect of brackets on the oral cavity soft tissues increases after their accidental breakage. Braces can be broken when chewing hard and dry food. The most likely accidental breakage of braces happens when chewing hard foods such as bones and "hard" pills (Figure 3 ).

After analyzing the results, we found out that braces which are mounted on the tooth rows do not cause damage to the lips and cheeks in the absence of chewing movements. In particular, soft tissue injuries in the oral cavity were not observed at night and during anesthesia. Lips and cheeks injuries are caused by continuous repetitive chewing movements. It is shown that local injuries of the lips and cheeks are caused not by the braces itself, but by the friction between their hard and hilly parts with the inner surface of the mouth. Therefore, we described this disease as "blistering disease of cheeks and lips". It is found that the appearance of local hyperthermia zone on the cheek and/or lip inner surface may be considered as a diagnostic symptom for the development of local inflammation. It is established that early diagnostics of blistering disease is impossible without thermal imaging.

In addition, for early diagnosis of blistering disease of cheeks and lips, we have developed a new infrared diagnostics technology. A new way of infrared diagnostics of early blistering disease of lips and cheeks caused by brackets is based on short-term pre-cooling of oral surfaces. Our results showed that preliminary intentional decrease of local temperature of lips and cheeks surface by several degrees Celsius intensifies temperature contrast between healthy and inflamed tissue areas and improves the accuracy of diagnosis of early blistering disease (Figure 4).

\section{Disscusion}

These results allow us to recommend infrared thermography for monitoring the dynamics of the local temperature in cheeks and lips during the first 4 days after the installation of braces. Infrared diagnostics of local hyperthermia zones at that side of the face, which patient mainly uses to chew food should be performed with particular care. It is proved that the use of a thermal imaging camera improves the quality of dental treatment during the installation 


\subsection{1/qirt.2016.139}

of braces, because it provides early detection of the blistering disease of cheeks and lips. Early diagnosis of the blistering disease prevents the development of blisters and ulcers, i.e. the development of dangerous complications of iatrogenic disease. In addition, thermal imaging camera allows us to shoot a film on the dynamics of the local temperature and the colour of facial skin and mucous membranes of the mouth, which, in its turn, can be archived using the flash memory, and can then be used by dentists as an additional document proving the quality of dental treatment provided to the patient.

\section{Figures}

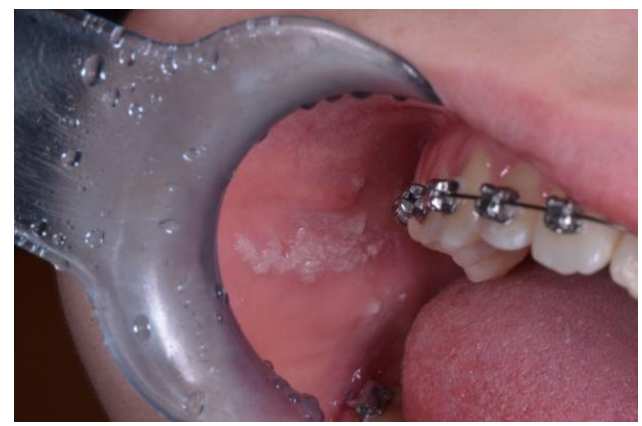

Fig. 1. Multiple blisters on the mucous membrane surface in the right cheek, 3 days after the brackets installation on the upper row of teeth (female $P$., 25 y.o.)

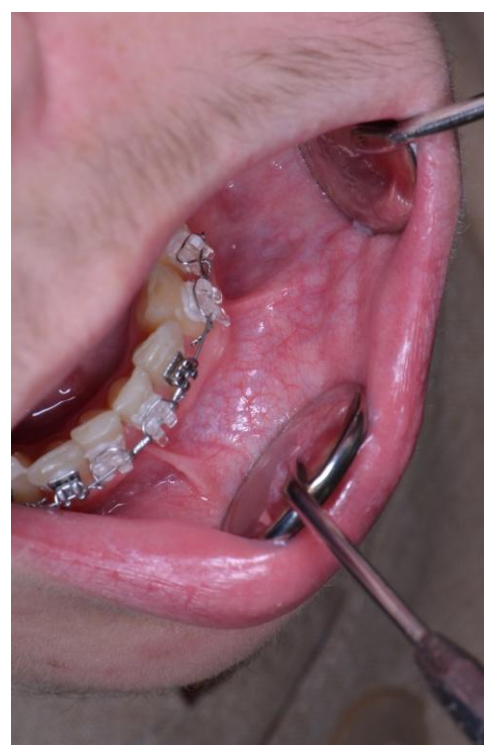

1 a

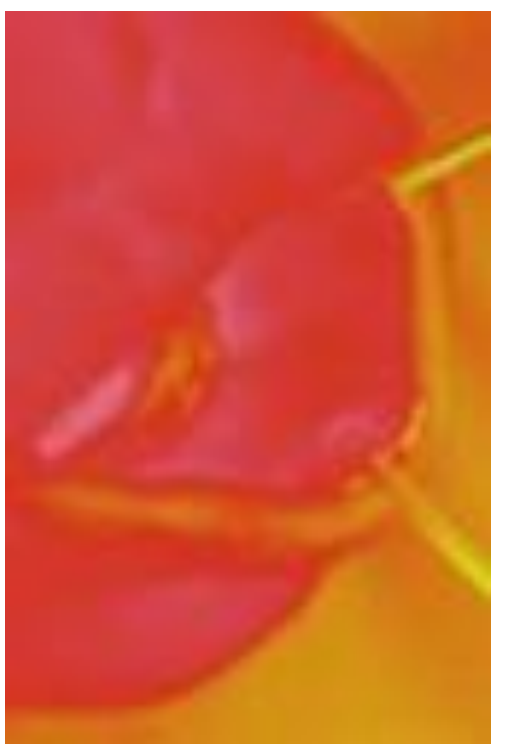

$1 \mathrm{~b}$

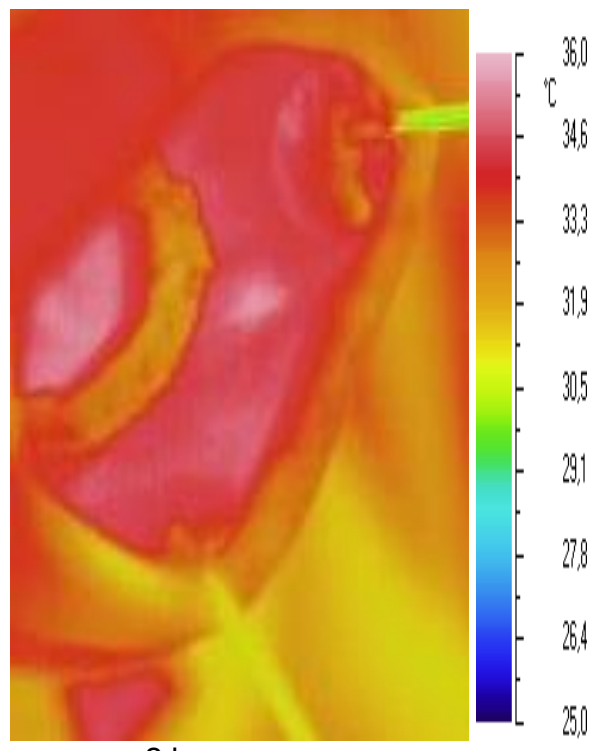

$2 \mathrm{~b}$

Fig. 2. The inner surface of the lower lip of the patient after the installation of braces: $1 A$ and $1 b-1$ minute after the installation of braces, $2 b-2$ days after the installation of braces. (patient $V .21$ years). Photo $2 b$ is seen a hotbed of local hyperthermia to the left of the center line.
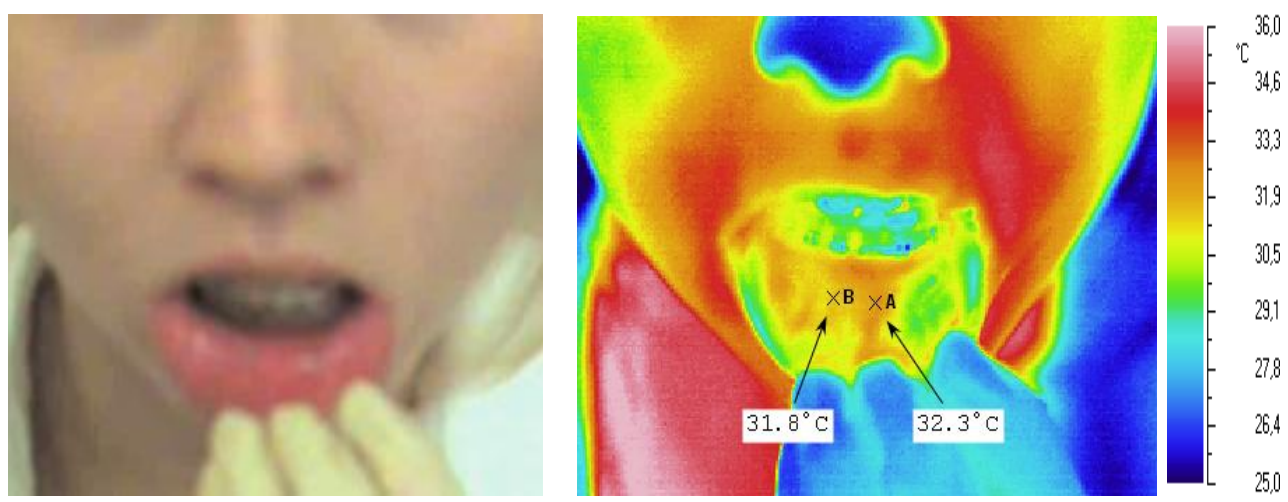
Fig. 3. Patient's lower lip inner surface in 3 days after the braces breakage caused by chewing metamizole sodium tablets. $A$ - in the visible radiation spectrum, $B$ - in the infrared spectrum. (Patient S., 22 y.o.)

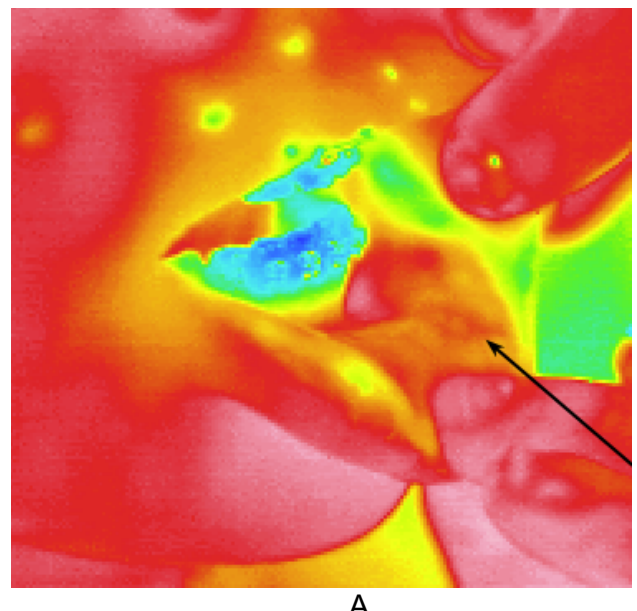

A

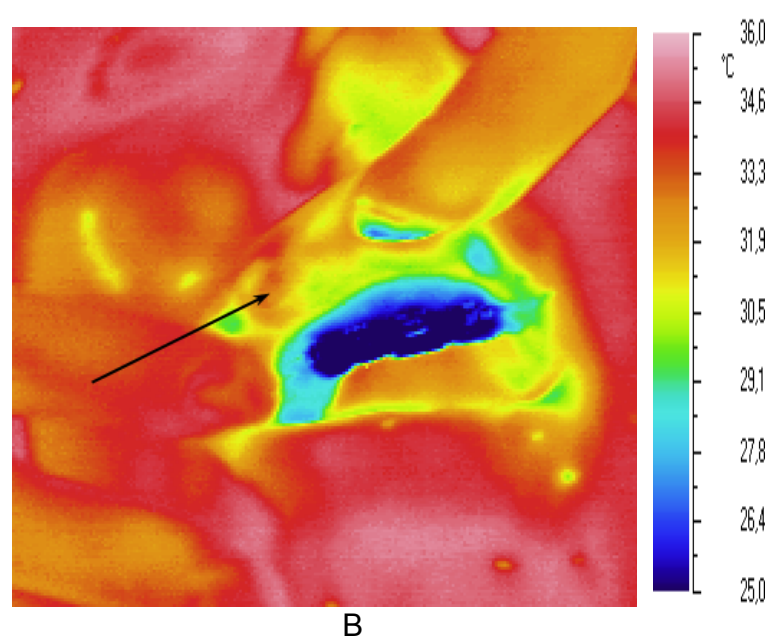

Fig. 4. Lips inner surface in 7 days after the installation of brackets on the lower row of teeth (A) (patient S., 42 y.o.) and on upper row of teeth (B) (patient. V. 48 y.o.). Note: The arrow indicates the local hyperthermia zone, detected by the thermal imaging camera

\section{REFERENCES}

[1] Urakov A.L., Reshetnikov A.P., Urakova N.A., et al., inventors. Method of protecting tissues of the corner of your mouth when remove tartar from the roots of wisdom teeth. Russian Federation patent RU 2357689. 2009 June 10.

[2] Urakov A.L., Reshetnikov A.P., Pozhilova E.V. Tablets as traumatic things to the mucous membranes, teeth and dental structures. Modern problems of science and education; 2, 2013. (URL: www.science-education.ru/1088480).

[3] Strelkov N.S., Urakov A.L., Urakova N.A., et al., inventors. Method for preventing demineralizing action solid dosage forms on the tooth enamel. Russian Federation application 2006141928. 2008 June 20.

[4] Urakov A.L., Strelkov N.S., Ivanova L.B., et al., inventors. Method of injecting after injecting drug infiltration. Russian Federation patent RU 2333001. 2008 September 10.

[5] Urakov A.L., Urakova N.A., Ivanova L.B., et al., inventors. Method of improving injection safety. Russian Federation patent RU 2340361. 2008 December 10.

[6] Urakov A.L., Reshetnikov A.P., Urakova N.A., et al., inventors. Method of protecting tissues of the corner of his mouth while removing tartar from the roots of wisdom teeth. Russian Federation patent RU 2357689. 2009 June 10.

[7] Reshetnikov A.P., Urakov A.L., Strelkov N.S. Way of restoration of the tooth. Russian Federation patent RU 2360640. 2009 July 10.

[8] Urakov AL., Urakova NA., Dement'ev VB., et al., inventors. Dental positioned. Russian Federation patent RU 2358641. 2009 June 20.

[9] Urakov A.L., Reshetnikov A.P., Reshetnikova A.A., et al., inventors. Method for splinting braces. Russian Federation patent RU 2437632. 2011 December 27.

[10] Urakov A.L., Ammer K., Urakova N.A., et al. Infrared thermography can discriminate the cause of skin discolourations. Thermology international; 25/4: pp. 209-215, 2015.

[11] Urakov A.L., Urakova N.A., Reshetnikov A.P., et al., inventors. Method of making and installing dental constructs. Russian Federation patent RU 2469640. 2012 December 20.

[12] Urakov A.L., Urakova N.A., Reshetnikov A.P., et al., inventors. Artificial food ball and method of use of artificial food bolus for rapid assessment of the patient's adaptation to the dental structure. Russian Federation patent RU 2533840. 2014 November 20.

[13] Reshetnikov A.P., Nikitiuk D.B., Urakov A.L., et al., inventors. Method of removing the soft tissue deficiency around the implant. Russian Federation patent RU 2558996. 2015 August 10.

[14] Reshetnikov A.P., Urakov A.L., Nikitiuk D.B. Method sinus lift with dental implants. Russian Federation patent RU 2563090. 2015 September 20.

[15] Urakov A.L., Urakova N.A., Nasyrov R.M., et al., inventors. Method infrared differential express-diagnostics of bruising and soft tissue injury. Russian Federation patent RU 2577510. 2016 March 20. 